Article

\title{
Efficiency and Spatial Equity Impacts of High-Speed Rail on the Central Plains Economic Region of China
}

\author{
Xiaomin Wang and Wenxin Zhang * \\ Faculty of Geographical Science, Beijing Normal University, Beijing 100875, China; \\ 201631170012@mail.bnu.edu.cn \\ * Correspondence: wzhang@bnu.edu.cn
}

Received: 4 April 2019; Accepted: 29 April 2019; Published: 5 May 2019

\begin{abstract}
Efficiency impacts can be assessed based on improvements in accessibility promoted by the high-speed rail (HSR) project, focusing mainly on major urban areas. Spatial equity impacts originate from changes in the distribution of accessibility levels observed across such cities. This study uses the weighted average travel time and coefficient of variation to explore the impact of HSR on efficiency and spatial equity within the Central Plains Economic Region (CPER) and to build an "efficiency-equity" model to identify optimal upgrading of conventional rail (CR) lines to improve the accessibility of the CPER and mitigate regional disparities. The results indicate that since the operation of HSR, accessibility levels across the CPER have improved by roughly $24.56 \%$, and the spatial distribution of gains has been uneven since the most significant improvements have occurred in HSR cities and large cities. Inequality in regional accessibility has increased by $28.12 \%$, creating a more heterogeneous pattern of accessibility. With goals of "efficiency" and "equity", an examination of the upgrading of $\mathrm{CR}$ as an approach shows that "Xinxiang-Jiaozuo" and "Xinxiang-Jiaozuo-Sanmenxia-Yuncheng" are the best upgraded lines for improving the efficiency of the CPER and limiting regional disparities. The results of this study serve as supportive information for the planning and construction of HSR lines and networks and for underdeveloped regional transport system policymaking.
\end{abstract}

Keywords: accessibility; efficiency impacts; spatial equity; high-speed rail (HSR)

\section{Introduction}

The high-speed rail (HSR) with operating speeds ranging from $200 \mathrm{~km} / \mathrm{h}$ to $350 \mathrm{~km} / \mathrm{h}$ serves as an important component of transportation systems. Benefits produced by the HSR transport infrastructure in cities and regions are frequently demonstrated by their accessibility [1,2], a term generally defined as the ability to reach a designated location in an appropriate amount of time and to rely on transportation facilities, depending on the mobility of a person and the opportunity to achieve goals through mobility [3]. The most direct influence of HSR is accessibility improvement [4-6]. In general, HSR transport networks can increase regional or national accessibility through "time-space convergence" or "corridor effects" [7,8]. HSR transport infrastructure shortens travel times and promotes the mobility of and interactions between people engaged in different economic activities, allowing individuals to travel more frequently and over longer distances [9]. Accessibility improvement plays an important role in restructuring spatial variation and new economic patterns observed across regions [10].

HSR drastically reduces the amount of time required to travel between cities, which has effects on efficiency and spatial equity. The accessibility improvements brought about by HSR are frequently viewed as efficiency implications [5,11]. The HSR transport infrastructure has obvious "time-space convergence" effects on a given region [12-14] and improves the overall accessibility of regional transportation networks. Particular cities with HSR stations of the HSR corridor may be turned into "regional cores" or "economic cores", whereas cities situated outside the HSR corridor may also gain 
accessibility benefits [5]. Another complementary and often contradictory manner involves the spatial distribution of these accessibility effects, which is equity $[15,16]$. HSR produces about unbalanced "time-space convergence", which increases the accessibility gap between regions and brings about transport equity. Acceptable levels of equal access can be guaranteed while ensuring maximum levels of accessibility and economic benefits from the HSR network when the improvement of the HSR infrastructure focuses on closing this gap.

Accessibility and the different effects of HSR have been widely studied in China and the rest of the world $[7,14,17,18]$, and several studies have been conducted on issues of efficiency and equity [2,5,19-21]. Currently, from the perspectives of efficiency and equity, consideration of equity issues has been applied to the transportation industry and regional policy documents in Europe, Japan, Spain and in other countries $[19,22,23]$. However, few studies have focused on addressing the issues of analysis of both the efficiency and spatial equity of the impacts of HSR operations. A summary table comparing the contributions of literature is provided in Table A1 of Appendix A.

In this context, the goal of this study is to quantify the efficiency and spatial equity impacts of HSR on the Central Plains Economic Region (CPER) of China from without HSR scenario to HSR scenario, and to build an "efficiency-equity" model to identify optimal upgrading of conventional rail (CR) lines to HSR (for cost reasons) for improving the accessibility of the CPER and achieving an equal distribution of accessibility across cities. In this study, we believe that the upgrading of key CR lines into HSR lines enable regional railway networks to balance efficiency and equity. The paper is structured as follows. Section 2 summarizes the existing literature analysing the efficiency and equity impacts of HSR. Section 3 provides detailed information on the case study area (the CPER) and on related research methods. Section 4 analyses the efficiency and spatial equity of the impacts of HSR operations and develops an "efficiency-equity" model to identify optimal upgrading for CR lines to accomplish the objective of enhancing accessibility and achieving an equal distribution of accessibility across cities. Finally, conclusions and a discussion are presented in Section 5.

\section{Review of the Impacts of HSR}

\subsection{Efficiency of HSR}

Efficiency impacts are evaluated in relation to improvements in accessibility resulting from an HSR project. Benefits resulting from HSR transport infrastructures in cities are commonly measured based on levels of accessibility $[1,5,11]$. This approach has been widely recognized in accessibility papers $[5,11,21,22,24]$. Since Hansen's (1959) seminal work, accessibility has become an important concept that has been widely applied to the fields of urban planning, transport geography, and other disciplines. The improvement of accessibility is a common goal of almost all transportation plans [25].

The investment and construction of an HSR system aims to improve transport efficiency levels, enhancing flows between cities and regions and increasing economic potential [23]. Therefore, the efficiency of HSR has been widely assessed by analysing accessibility levels $[1,26]$. The operation of an HSR system greatly reduces travel times between cities and enhances spatial interactions between cities and regions, thus, there is an obvious space-time convergence effect on a given region and significant improvement on the overall accessibility efficiency of regional land transport networks $[6,23]$. Gutiérrez et al. (1996) used a weighted average distance indicator to evaluate the accessibility impacts of the future European HSR network by comparing the current systems with the outlined plan for 2010. They found that Europe was shrinking because the distances covered over the length of time generally accepted for daytime travel (240 $\mathrm{min}$ to $300 \mathrm{~min}$ at most) have increased to as much as $1000 \mathrm{~km}$ to $1200 \mathrm{~km}$ [22]. Gutiérrez et al. (2010) further found that European HSR has shortened inter-city travel times, enabling the space-time convergence effect and significantly improving city accessibility levels and changing the existing regional spatial structure [12]. Kim measured the accessibility of each stage of HSR network extension in South Korea from 2004 to 2018 and concluded that South Korea cities and counties have become more accessible to one another by approximately $13 \%$ in terms of travel-time 
savings and by $11 \%$ in terms of increasing attractiveness and that the accessibility and attractiveness of the studied cities have been improved overall [2].

As HSR construction and operation costs are enormous, the HSR infrastructure is mostly concentrated in regions with dense populations and developed economies. According to the expanding scope of new HSR projects and their services, particular cities with HSR stations of an HSR corridor can experience direct effects, promoting further changes in land use and economic activity around this corridor and potentially turning such areas into "regional cores" or "economic corridors" [5,23], whereas cities situated outside the HSR corridor may also gain accessibility benefits. Accessibility is required for cities to enhance their competitiveness, but the relationship between connectivity and economic growth is complex and uncertain [2,5].

In summary, most studies have found that major improvements in accessibility levels have been limited to large cities and cities positioned within HSR corridors due to locational and geographical advantages derived from HSR services [1,27]. This encourages macroeconomic or microeconomic activities relative to a given location as well as changes in the distribution of economic factors, which can compromise common goals for equity in the transport infrastructure development.

\subsection{Spatial Equity of HSR}

The concept of equity is related to the distribution of a given effect. Equity in transport seems to be a very complex phenomenon resulting from the fact that there is no standard definition of the term [28]. Common practice typically identifies two main dimensions: Vertical and horizontal [29]. Vertical equity refers to disparities between individuals and groups with different capacities and needs [30]. The horizontal dimension refers to spatial disparities emphasizing the importance of a uniform distribution of accessibility levels across geographical space areas [31-33]. In this study, efficiency impacts can be assessed based on improvements in accessibility promoted by an HSR project, and spatial equity impacts originate from changes made in the distribution of accessibility levels across cities.

The assessment of the equity impacts of HSR is frequently performed by using spatial impact models supported by a GIS and the results of accessibility assessments, which are compared to the spatial distributions of CR $[5,12,19,21,34]$. Relevant works have proven the effectiveness of employing accessibility or "equity of access" as the main input variable for assessing equity impacts and have proven capacity to address both efficiency and equity issues when planning HSR operations $[19,20]$. Early examples of the use of accessibility to evaluate spatial equity impacts can be traced to the late 1970s. For instance, Domanski (1979) explored the spatial of accessibility of concentration and dispersion [35].

The debate on the effects of HSR on the equity of core cities, marginal cities and sparsely populated regions; HSR and non-HSR cities; and cities of different sizes remains on the research agenda. The results of several studies show that the operation of HSR can have an imbalanced space-time convergence effect, aggravating the difference in accessibility between regions and producing problems of transport inequity $[2,5,36]$. Levinson conducted a western HSR accessibility study and found that small and medium-sized cities may achieve absolute gains in accessibility (absolute accessibility), they may lose in relative position, as central cities accumulated a greater share of the now larger total accessibility (relative accessibility), thus showing that HSR construction exacerbates regional differences in accessibility [17]. Sánchez-Mateos and Givoni measured the potential accessibility impacts of a new HSR line in the UK by means of the rail travel times to London. The authors argue that from the point of view of geographic spread, the accessibility benefits from the HSR line have been relatively limited. While travel times have been significantly shortened for cities at the end of the line, minimal travel time savings have been enjoyed in cities located in the "middle" of the line [20]. Hall noted that the spatial impact of the new HSR line has been relatively complex. While it has benefitted large-scale central cities connected to the new lines and especially urban centres, it may also threaten the location advantages of peripheral cities [37]. In contrast, some results show that high-speed transportation facilities (including highways and high-speed railways) can significantly improve the accessibility and location advantages of sparsely populated or isolated areas and enhance 
regional equity [38]. Some scholars have argued that whether regional accessibility changes caused by HSR are spatially uniform depends on the spatial scales used by researchers but that a change in accessibility should inevitably lead to a game between HSR and other modes of transportation (mainly CR and airline) $[4,39,40]$.

The objective debate on "efficiency and equity" concludes that transport planners and decisionmakers should synchronously integrate the compatibility of two objectives: The improvement of accessibility and the achievement of an equal distribution of accessibility across cities and regions. From a review of the impacts of HSR, we found that related studies have only measured the inequality of accessibility while providing some policy recommendations but have not addressed issues surrounding efficiency and equity by outlining a reasonable approach. Spatial unequal has serious consequences such as spatial polarization of region, the formation of economic differences, envy of the wealthy and social exclusion. The situation goes against the sustainable development of the city and furthermore, imposes restrictions on the sustainable development of social economy of the country and the regions. When the construction of an HSR system does not mitigate regional differences to achieve integration goals, both infrastructure and suitable service regulatory frameworks are needed to achieve such goals, and effective measures must be applied at the local level [41].

Previous studies have demonstrated that HSR networks are regarded as important tools for limiting regional differences and promoting regional integration [42]. According to Monzón et al. (2019) spatial equity can include enhancing the connectivity of HSR networks and reducing the risk of polarization, reducing disparities in the territorial distribution of accessibility, achieving higher levels of territorial cohesion in the distribution of accessibility [43]. Considering the efficiency and construction costs of HSR lines, the upgrading of CR and semi-HSR lines has been regarded as a means of both increasing efficiency and relieving spatial disparities of accessibility within developing regions $[2,5,21]$. Wu et al. recommended that CR lines, which are much cheaper than HSR lines, be upgraded in underdeveloped areas of China, as the value of time remains low for most residents [44]. HSR stations upgraded from CR stations are easy for most residents to access as after decades of development, CR stations are typically integrated into urban areas and have become a part of the cities in which they are built [21]. Upgraded CR lines and stations can relieve pressures on intra- and extra-city accessibility to HSR services.

\section{Setting, Data, and Methodology}

\subsection{Study Area}

The CPER is one of the most important components of China's Major Function Oriented Zoning system [45]. It is a functional region based on urban agglomeration planning in China located in central China along the central and lower reaches of the Yellow River. Its geographical position is of significant importance, and its market presents great potential. Overall, the CPER is a relatively less developed region in China. The "Zheng-Bian-Luo" (Zhengzhou-Kaifeng-Luoyang) metropolitan area is a core urban area and the economic powerhouse of the CPER. Its administrative division covers a total area of $289,000 \mathrm{~km}^{2}$. This area includes Henan Province and its surrounding area as well as 30 cities and three counties, including all 18 cities in the Henan Province; 3 cities (Changzhi, Yuncheng, and Jincheng) in the Shanxi Province; 2 cities (Xingtai and Handan) in the Hebei Province; 3 cities (Liaocheng, Heze, and Dongping Taian) in the Shandong Province; and the Huaibei, Bengbu, Fuyang, Suzhou, Bozhou, Panji districts and Fengtai county of Huainan, Anhui Province (Dongping, Taian, Panji, and Fengtai are included in this study). The classification used for the cities is shown in Table 1. 
Table 1. City classification for the CPER.

\begin{tabular}{ccl}
\hline \multicolumn{1}{c}{ Category } & Number of Cities & \multicolumn{1}{c}{ City } \\
\hline With HSR cities & 17 & $\begin{array}{l}\text { Zhengzhou; Kaifeng; Luoyang; Anyang; Shangqiu; } \\
\text { Xinyang; Jiaozuo; Luohe; Xinxiang; Xuchang; } \\
\text { Zhumadian; Hebi; Sanmenxia; Xingtai; Handan; } \\
\text { Suzhou; Bengbu }\end{array}$ \\
\hline Without HSR cities & 13 & $\begin{array}{l}\text { Zhoukou; Nanyang; Jiyuan; Pingdingshan; Puyang; } \\
\text { Yuncheng; Jincheng; Changzhi; Liaocheng; Heze; } \\
\text { Huaibei; Bozhou; Fuyang }\end{array}$ \\
\hline $\begin{array}{c}\text { Large cities }\left(50 \times 10^{4}<\text { Urban }\right. \\
\left.\text { population }<100 \times 10^{4}\right)\end{array}$ & 9 & $\begin{array}{l}\text { Zhengzhou; Kaifeng; Luoyang; Anyang; Jiaozuo; } \\
\text { Xinxiang; Pingdingshan; Handan; Huaibei }\end{array}$ \\
\hline $\begin{array}{c}\text { Small to medium-sized cities } \\
\left(0<\text { Urban population }<50 \times 10^{4}\right)\end{array}$ & 21 & $\begin{array}{l}\text { Zhoukou; Shangqiu; Nanyang; Xinyang; Jiyuan; } \\
\text { Luohe; Xuchang; Zhumadian; Hebi; Puyang; } \\
\text { Sanmenxia; Yuncheng; Jincheng; Changzhi; Xingtai; } \\
\text { Liaocheng; Heze; Suzhou; Bengbu Bozhou; Fuyang }\end{array}$ \\
\hline
\end{tabular}

According to the Mid-to-Long Term Railway Network Plan (2008), the China HSR grid built "The Four Vertical and Four Horizontal HSR framework" consisting of eight HSR corridors-four running north-south and four going east-west (known as $4+4$ ). With its geographical advantage, the CPER has assumed a leading role in the development of China's HSR and has built an HSR network quite rapidly (Figure 1). The Zhengxi (Zhengzhou-Xian) HSR completed in 2010 was the first HSR built in the CPER. Several lines have since been opened and been put into operation (Table 2), and there are now 17 cities along the HSR corridor. In this study, 17 cities along seven HSR lines and 13 non-HSR cities of the CPER were selected as research objects.

Table 2. High-speed rail (HSR) network extensions of the CPER.

\begin{tabular}{ccc}
\hline Operating Date & HSR & Site City \\
\hline $2010-1-28$ & Zheng-Xi HSR & Zhengzhou; Luoyang; Sanmenxia \\
\hline $2011-6-30$ & Jing-Hu HSR & Suzhou; Bengbu \\
\hline $2012-9-28$ & Shi-Wu HSR (Zhengzhou-Wuhan) & Xuchang; Zhumadian; Luohe; Xinyang \\
\hline $2012-12-26$ & $\begin{array}{c}\text { Shi-Wu HSR } \\
\text { (Shijiazhuang-Zhengzhou) }\end{array}$ & Xingtai; Hebi; Xinxiang; Anyang; Handan \\
\hline $2014-12-28$ & Zheng-Kai Intercity HSR & Kaifeng \\
\hline $2015-6-26$ & Zheng-Jiao Intercity HSR & Jiaozuo \\
\hline $2016-9-10$ & Zheng-xu HSR & Shangqiu \\
\hline
\end{tabular}




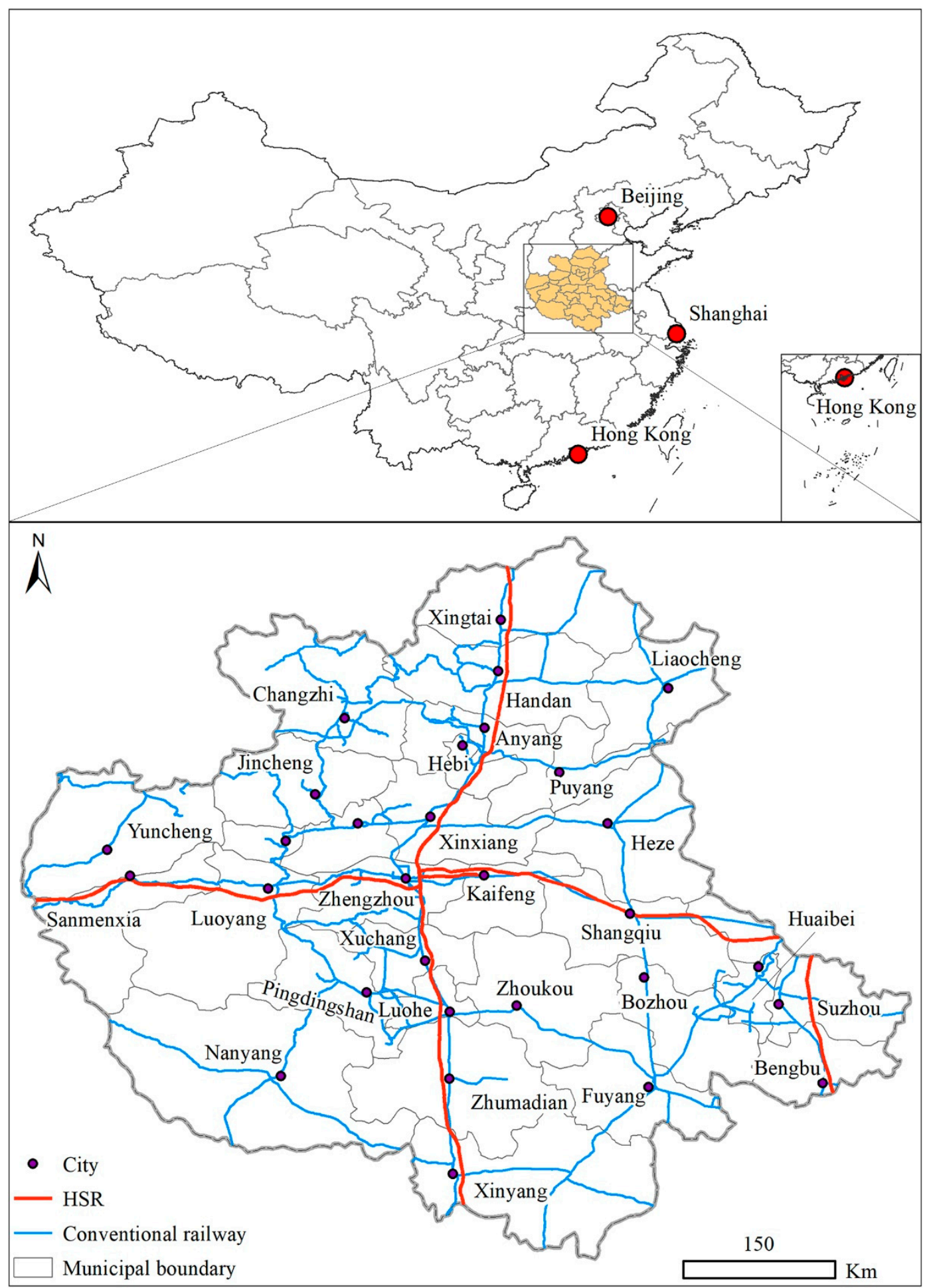

Figure 1. Railway network in Central Plains Economic Region (CPER) of China.

\subsection{Data}

For this study, 30 cities in the CPER were selected as research objects. As data, we measured the shortest travel times for travelling by rail between 30 cities and population. The shortest travel time was extracted from JPSKB (Ji Pin Lie Che Timetable) issued by Beijing Need for Time Technology Co., Ltd (No. 9 Manting Fangyuan Community, Qingyunli, Haidian District, Beijing). Transfer travel time data were obtained from Train timetables and Ctrip (www.12306.cn and www.ctrip.com). Seven types 
of train service are offered (see Table 3). P, K, T, Z are CR. D, C, G are HSR. Population values were obtained from the 2016 China Urban Statistical Yearbook.

Table 3. CR and HSR passenger train services.

\begin{tabular}{cccccccc}
\hline $\begin{array}{c}\text { Train Service } \\
\text { Type }\end{array}$ & $\mathbf{P}$ & $\mathbf{K}$ & $\mathbf{T}$ & $\mathbf{Z}$ & $\mathbf{D}$ & $\mathbf{C}$ & $\mathbf{G}$ \\
\hline Description & $\begin{array}{c}\text { General } \\
\text { train }\end{array}$ & Fast train & $\begin{array}{c}\text { Express } \\
\text { train }\end{array}$ & $\begin{array}{c}\text { Direct exp. } \\
\text { train }\end{array}$ & CRH train & Intercity-HSR & HSR train \\
\hline $\begin{array}{c}\text { Maximum speed } \\
(\mathrm{km} / \mathrm{h})\end{array}$ & 100 & 120 & 140 & 160 & {$[200,250]$} & 300 & {$[300,350]$} \\
\hline Practical speed & \multicolumn{70}{c}{$[60,120]$} \\
\hline
\end{tabular}

\subsection{Methodology}

\subsubsection{Weighted Average Travel Time}

Scholars commonly use three accessibility indicators: The weighted average travel time (WATT), economic potential, and daily accessibility $[1,8,22,24,46-48]$. HSR is characterized by high travel speeds, and travel time is assumed to be particularly important [20]. This study explores the impact of HSR on efficiency and therefore the WATT is used as a suitable indicator for measuring accessibility in this work.

The WATT is based on the average travel time with the scale of the location used as the weight in this indicator. The WATT can be described in the following manner: The lower the score is, the higher the level of accessibility of a node and the closer its contact to other locations in the region; the reverse is also true. Its mathematical expression is as follows:

$$
T_{i}=\frac{\sum_{j}^{j \neq i} M_{j} \cdot t_{i j}}{\sum_{j}^{j \neq i} M_{j}}
$$

where $T_{i}$ represents the accessibility of location $i, t_{i j}$ represents the shortest travel time to destination $j$ by querying and $M_{j}$ represents the mass of destination city $j$, which is measured from the population and GDP of the destination (in our case, this is the total population of each city).

\subsubsection{Coefficients of Variation}

The coefficient of variation (CV) index evaluates the degrees of spatial variation in accessibility across cities and helps to understand the trend of accessibility disparity with HSR and without HSR. This index has been previously used for this purpose in similar studies $[2,5,19]$. The Equation for the $\mathrm{CV}$ is:

$$
\mathrm{CV}=\frac{\sigma^{P}}{\sum T_{i} M_{i} / \sum M_{i}}
$$

where $\mathrm{CV}$ represents the coefficient of variation for the scenario, $\sigma^{P}$ represents the standard deviation of accessibility values $T_{i}$, and $M_{i}$ represents the weight of the total population. The CV index can be described in the following manner: An increase in CV indicates that the difference in accessibility between urban areas has gradually increased and that spatial polarization is strong, while a decrease in $\mathrm{CV}$ indicates that the difference in accessibility between cities has gradually decreased and that spatial diffusion is strong [5].

\subsubsection{Constructed "Efficiency-Equity" Model}

It is argued that HSR can play a role in accomplishing this more balanced and sustainable development of cities and region, providing opportunities for an interconnected region, with the benefit of promoting regional economic integration $[16,36]$. Solutions can be improved by synchronously 
integrating the maximization of two objectives: The improvement of accessibility and the achievement of an equal distribution of accessibility across cities, thus promoting regional sustainable development. First, it is necessary to filter the data. Before upgrading, the shortest travel times between each pair of cities among the 30 cities studied were collected from JPSKB. The shortest amount of travel time by rail between two cities can involve the use of a direct train (i.e., a train without transfer) or the use of a train that includes transfer. When the shortest travel time between two cities, according to the JPSKB, involves a direct CR train, this section of the CR line can be upgraded to an HSR system, as it is difficult to calculate travel times after upgrading the CR line that includes transfer in our model. In addition, the fact is that the travel time by rail from city $i$ to city $j$ approximately equals to that from city $j$ to city $i$. For the convenience of calculation, we assume that both of them are equal to each other.

Second, we construct an "efficiency-equity" model as follows. (1) The speed of CR trains varies from $60 \mathrm{~km} / \mathrm{h}$ to $120 \mathrm{~km} / \mathrm{h}$ from Train timetables depending on the number of stops the travel contains. In this paper, we use the average travel speed of different types of CR train services to determine rail travel time in the "efficiency-equity" model. The average speed of conventional railways was set at $90 \mathrm{~km} / \mathrm{h}$. (2) We set the speed of HSR upgraded from CR to $250 \mathrm{~km} / \mathrm{h}$. Two types of HSR train systems are employed in China. The first mode involves upgrading CR lines, thus they can operate much cheaper HSR services at speeds of $200-250 \mathrm{~km} / \mathrm{h}$. The second mode involves a more advanced system supporting travel speeds of $300-350 \mathrm{~km} / \mathrm{h}$. Based on China's advanced upgrading technologies for wheel-track trains and operation costs of the HSR line, we choose to upgrade CR lines, which can support less expensive HSR services at a speed of $250 \mathrm{~km} / \mathrm{h}$. Similar HSR services are employed in Italy and Sweden and for a few lines in China. (3) After a CR line is upgraded to an HSR line, a onetime transfer of $30 \mathrm{~min}$ is added when the travel time of the two cities is equal to the sum of the travel time of each line. All transfer times were obtained from official train timetables (Train timetables and Ctrip) and the shortest is roughly $30 \mathrm{~min}$, and thus based on the principle of the shortest travel time, we adopt the time of $30 \mathrm{~min}$. (4) When the newly upgraded section of an HSR is passed, the principle of the shortest travel time can be calculated with $A C=A B+B C+t$. The travel times of rail lines travelling between cities that do not pass through the newly upgraded HSR line can only be expressed as the original time. Therefore, the shortest travel time between two cities is the shortest period of all possible routes. (5) By upgrading one line (or two lines) of CR to HSR, the accessibility of the CPER increases significantly, and the CV decreases the most.

In an undirected weighted graph $G=(V, E, W), G$ consists of vertices, edge sets and edge weights where $V$ sets represent cities and $E$ sets represent the shortest travel time required to travel from one city to another before upgrading. The travel time between cities varies depending on the different rail lines involved. $W$ sets represent the weights of the rail lines.

$$
V=\left\{v_{i}, v_{j} \mid i, j=1,2,3, \ldots, n\right\}, E=\left\{e_{i j} \mid i, j=1,2,3, \ldots, n\right\}, W=\left\{w_{i j} \mid i, j=1,2,3, \ldots, n\right\}, n \text { denotes the }
$$
number of cities. When there is a rail line between city $v_{i}$ and city $v_{j}$ before upgrading, then $w_{i j}=1$, and the travel time is $w_{i j} \cdot e_{i j}$. Otherwise, when a rail line between $v_{i}$ and $v_{j}$ is upgraded to an HSR line, then $w_{i j}=9 / 25$. When there is a route between $v_{i}$ and $v_{j}$ not through the upgraded line, the travel time of this route $\left.R<v_{i}, v_{j}\right\rangle$ can be defined by $\left.R<v_{i} \rightarrow v_{j}\right\rangle=w_{i j} \cdot e_{i j}$. When there is a route from $v_{i}$ to $v_{j}$ through an upgraded line and should take a transfer only at node $k$, then the travel time of this route $R<v_{i}, v_{j}>$ can be defined by $R<v_{i} \rightarrow v_{k} \rightarrow v_{j}>=w_{i k} \cdot e_{i k}+w_{k j} \cdot e_{k j}+t$. Therefore, the route running from $v_{i}$ to $v_{j}$ includes $R<v_{i} \rightarrow v_{j}>$ and $R<v_{i} \rightarrow v_{k} \rightarrow v_{j}>$, as shown in Figure 2. $t$ is the transfer time, which is a constant. When the route from $v_{i}$ to $v_{j}$ should take transfer at $p$ cities, then the transfer time is $p \cdot t$. 


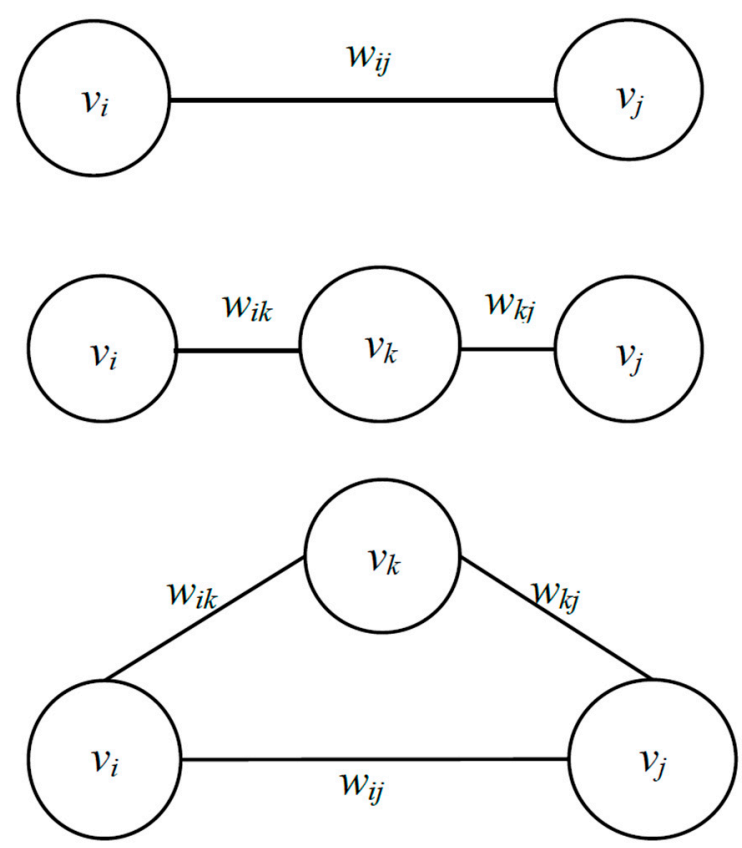

Figure 2. Potential routes between cities.

When considering upgraded lines, we assume that from one origin (city) to a destination (city) via upgraded lines of a rail transport network, there are various routes from $v_{i}$ to $v_{j}$, and we select the shortest route depending on the shortest travel time, which is defined as $\mathrm{R}\left(v_{i}, v_{j}\right)=\operatorname{Min} R<v_{i}, v_{j}>$.

When considering edges $e \in E$ to upgrade, which will change weights $w$, then the shortest travel time route $R\left(v_{i}, v_{j}\right)$ may be changed. We then obtain each city $v_{i}{ }^{\prime}$ S WATT $R\left(v_{i}\right)$ from Equation (3):

$$
R\left(v_{i}\right)=\frac{\sum_{j}^{j \neq i} M_{j} \cdot R\left(v_{i}, \mathrm{v}_{j}\right)}{\sum_{j}^{j \neq i} M_{j}}
$$

According to each city's WATT, we can calculate CV values of the whole CPER based on different scenarios of the CR line upgrading, and then find out the CR line with the most CV decrease after upgrading. To calculate the shortest routes based on travel times between cities, we establish the following two models:

Model 1:

Only one CR line is upgraded. We assume cities $v_{i}, v_{j}, v_{b}$, and $v_{c}$ are connected to one another. $e$ and $w$ represent connections and time weights between cities, respectively.

Let $e_{b c}$ be the upgraded line. Then, $w_{b c}=9 / 25$, and when $(i, j) \neq(b, c), w_{i j}=1$. $R$ denotes routes among vertices. Three scenarios are considered:

Scenario 1: Origins and destinations are excluded from upgraded lines, $i, j \neq b, c$ and there are three routes from $v_{i}$ to $v_{j}: R_{1}=e_{i b}+w_{b c} \cdot e_{b c}+e_{c j}+2 t, R_{2}=e_{i c}+w_{b c} \cdot e_{c b}+e_{b j}+2 t, R_{3}=e_{i j} . w_{i j}$. The shortest-travel-time route is $R\left(v_{i}, v_{j}\right)=\operatorname{Min}\left\{R_{1}, R_{2}, R_{3}\right\}$ as shown in Figure 3a.

Scenario 2: One node from an origin and destination is located on an upgraded line. For instance, when $i=b, j \neq b, c$, there are two routes from $v_{i}$ to $v_{j}: R_{1}=w_{i c} \cdot e_{i c}+e_{c j}+t, R_{2}=e_{i j}$. The shortest-travel-time route is $R\left(v_{i}, v_{j}\right)=\operatorname{Min}\left\{R_{1}, R_{2}\right\}$.

Scenario 3: Both origins and destinations are located on an upgraded line. For example, $i=b, c=j$, and the shortest-travel-time route is $R\left(v_{i}, v_{j}\right)=w_{i j} \cdot e_{i j}$. 
(a)

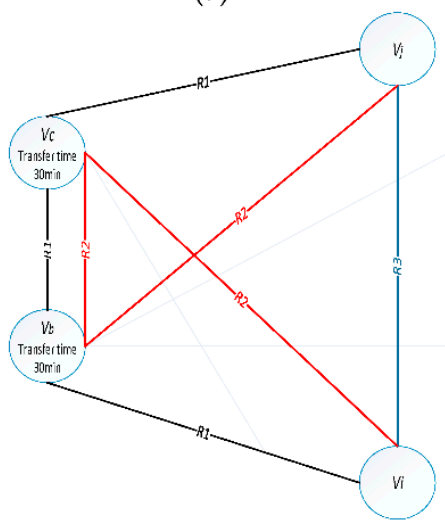

(b)

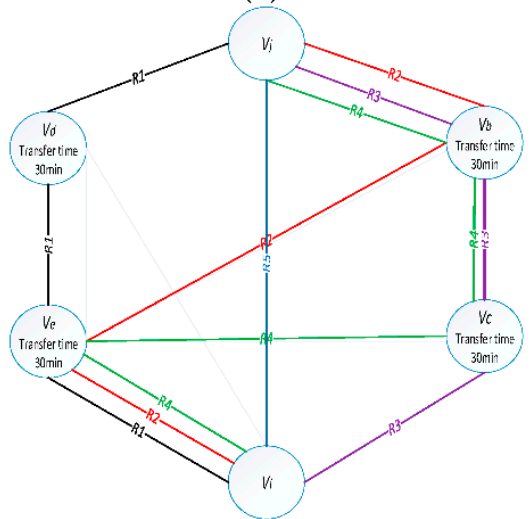

Figure 3. Schematic diagram of the model. (a) Model 1; (b) Model 2.

Model 2:

We assume that cities $v_{i}, v_{j}, v_{b}, v_{c}, v_{d}$, and $v_{e}$ are connected to one another. There are two CR lines to upgrade. We randomly select two lines to upgrade such as $e_{b c}$ and $e_{d e}$, and then $w_{i j}=1$ when $(i, j) \neq$ $(b, c),(d, e)$, and $w_{i j}=9 / 25$ when $(i, j)=(b, c)$ or $(d, e)$. Two scenarios are considered.

Scenario 1: We randomly select two lines to upgrade $e_{b c}$ and $e_{d e}$, which are not connected to, which means that $b \neq c \neq d \neq e$. Between various origins and destinations, there are different routes through two upgraded lines. When considering the relationships between origins, destinations and upgraded lines, four outcomes can result.

(1) Origins and destinations are excluded from upgraded lines, $i, j \neq b, c, d, e$. From $v_{i}$ to $v_{j}: R_{1}=$ $e_{i d}+w_{d e} \cdot e_{d e}+e_{e j}+2 t, R_{2}=e_{i e}+w_{d e} \cdot e_{e d}+e_{d j}+2 t$, and $R_{3}=e_{i c}+w_{b c} \cdot e_{c b}+e_{b j}+2 t \ldots 13$ routes are available. The shortest-travel-time route is $R\left(v_{i}, v_{j}\right)=\operatorname{Min}\left\{R_{1}, R_{2}, \ldots, R_{13}\right\}$. Since there are too many routes, only five of them are shown in the Figure $3 b$.

(2) One node of origins and destinations is located on one upgraded line. For instance, $i \neq b, c, d, e$ and $j=$ e. From $A_{i}$ to $A_{j}: R_{1}=e_{i d}+w_{d j} \cdot e_{d j}+t, R_{2}=e_{i b}+w_{b c} \cdot e_{b c}+e_{c d}+w_{d j} \cdot e_{d j}+3 t$, and $R_{3}=e_{i b}+$ $w_{b c} \cdot e_{b c}+e_{c j}+2 t \ldots$ and six routes are available. $R\left(v_{i}, v_{j}\right)=\operatorname{Min}\left\{R_{1}, R_{2} \ldots, R_{6}\right\}$.

(3) Origins and destinations are positioned on two separate upgraded lines. For example, $i=b$, $j=d$. From $A_{i}$ to $A_{j}: R_{1}=e_{i j}, R_{2}=w_{i c} \cdot e_{i c}+e_{c j}+t, R_{3}=w_{i c} \cdot e_{i c}+e_{c e}+e_{e j}+2 t$, and $R_{4}=e_{i e}+w_{e j} \cdot e_{e j}+t$. $\mathrm{R}\left(v_{i}, v_{j}\right)=\operatorname{Min}\left\{R_{1}, R_{2}, R_{3}, R_{4}\right\}$.

(4) Origins and destinations are positioned on the same upgraded lines. For example, $i=d, j=e$. From $v_{i}$ to $v_{j}: R_{1}=w_{i j} \cdot e_{i j}, R_{2}=e_{i b}+w_{b c} \cdot e_{b c}+e_{c j}+2 t$, and $R_{3}=e_{i c}+w_{b c} \cdot e_{c b}+e_{b j}+2 t . R\left(v_{i}, v_{j}\right)=$ $\operatorname{Min}\left\{R_{1}, R_{2}, R_{3}\right\}$.

Scenario 2: $e_{b c}$ and $e_{d e}$ are selected as upgraded lines, and they are connected, for example $c=e, b$ $\neq d$. The following five outcomes can result.

(1) Origins and destinations are excluded from upgraded lines. $i \neq j \neq b \neq c \neq d$. From $v_{i}$ to $v_{j}: R_{1}=$ $v_{i j}, R_{2}=v_{i b}+w_{b c} \cdot e_{b c}+e_{c d}+e_{d j}+3 t$, and $R_{3}=e_{i d}+w_{d e} \cdot e_{d c}+w_{b c} \cdot e_{c b}+e_{b j}+3 t \ldots$ seven routes are available. $R\left(v_{i}, v_{j}\right)=\operatorname{Min}\left\{R_{1}, R_{2} \ldots, R_{7}\right\}$

(2) One node from origins and destinations is located on one upgraded line, not at the junction of the two upgraded lines; the other node is not on the two upgraded lines. For example, $i \neq b, c, d$ and $j=d$. From $v_{i}$ to $v_{j}: R_{1}=e_{i d}, R_{2}=e_{i c}+w_{c j} \cdot e_{c j}+t$, and $R_{3}=e_{i b}+w_{b c} \cdot e_{b c}+w_{c j} \cdot e_{c j}+2 t, R\left(v_{i}, v_{j}\right)=$ $\operatorname{Min}\left\{R_{1}, R_{2}, R_{3}\right\}$.

(3) One node from origins or destinations is located at the junction between two upgraded lines. For example, $i \neq b, c, d$ and $j=c$. From $v_{i}$ to $v_{j}: R_{1}=e_{i j}, R_{2}=e_{i b}+w_{b j} \cdot e_{b j}+t, R_{3}=e_{i d}+w_{d j} \cdot e_{d j}+t$, and $R\left(v_{i}, v_{j}\right)=\operatorname{Min}\left\{R_{1}, R_{2}, R_{3}\right\}$. 
(4) Origins and destinations are located along two upgraded lines and both of them are not at the junction. For example, $i=b, j=d$. From $A_{i}$ to $A_{j}: R_{1}=e_{i j}, R_{2}=w_{i c} \cdot e_{i c}+w_{c j} \cdot e_{c j}+t$, and $\mathrm{R}\left(v_{i}, v_{j}\right)=$ $\operatorname{Min}\left\{R_{1}, R_{2}\right\}$.

(5) Origins and destinations are located on two upgraded lines, and one is located at the junction. For example, $i=b, j=c . R_{1}=w_{i j} \cdot e_{i j}, R_{2}=e_{i d}+w_{d j} \cdot e_{d j}+t$, and $R\left(v_{i}, v_{j}\right)=\operatorname{Min}\left\{R_{1}, R_{2}\right\}$.

We compare travel times for possible routes for the above cases to obtain the shortest travel time for each case. A schematic diagram of the model is shown in Figure 3. The above process is applied using the Python software.

\section{Results and Analysis}

\subsection{Efficiency Analysis}

The shortest travel time between $30 \times 30$ cities was determined, and a time weight matrix was obtained. The WATT values were calculated for all CPER cities included in the study to estimate efficiency impacts with and without HSR networks in the CPER. Then, according to the WATT value and using the inverse distance weighted interpolation method, we obtained a spatial pattern and change map for the accessibility of the CPER before and after HSR operation. After the HSR operation in the CPER, the WATT values of all the cities were shortened (Table 4), and accessibility in the CPER improved significantly. In total, 30 cities showed improved access to one another, and cities became more interconnected. Consequently, time and space convergence effects are obvious. As shown in Figure 4, the WATT values for cities in the CPER was measured as $345.78 \mathrm{~min}$ and $260.85 \mathrm{~min}$ before and after the introduction of the HSR, respectively, revealing a reduction rate of $24.56 \%$ in terms of travel time savings.

Absolute and relative changes in the WATT values before and after the introduction of the HSR were calculated. Cities showing a greater absolute increase in accessibility were concentrated along the HSR corridor. According to Table 4 and Figure 4, HSR has improved regional accessibility as a whole but with obvious spatial variations. Relative to those prior to the HSR introduction, regional WATT values were reduced by an average of $84.93 \mathrm{~min}$, and there are 14 cities whose WATT reductions are higher than the average decrease of the CPER. These cities that obtain a considerable improvement were mainly concentrated along HSR lines (12), just two of them are not along the HSR (2). Cities with low original accessibility level in the non-HSR scenario among these 14 cities obtain a considerable improvement after the introduction of the HSR. This situation occurs in Suzhou, Yuncheng, Sanmenxia, Bengbu, Xingtai, Huaibei. Due to their poor initial position, their absolute improvement is very high. Cities with high accessibility level in the non-HSR scenario among these 14 cities also obtain a considerable improvement after the introduction of the HSR, such as Luoyang, shangqiu. This situation is due to the fact that these cities already have good conventional railway connections. Thus, the operation of HSR has once again caused these cities to experience a considerable improvement in accessibility.

Cities showing a larger relative increase in accessibility are mostly located along the "HSR corridor". Relative to the scenario involving without HSR (as shown in Figure 4d), HSR cities experiencing high increases in accessibility include Suzhou, Sanmenxia, Xinyang, Zhumadian, Xingtai, Handan, Luoyang, Hebi, Aayang, Bengbu. Non-HSR cities located adjacent to HSR cities exhibit a secondary increase as they become beneficiaries of HSR "diffusion effects". These cities include Yuncheng adjacent to Sanmenxia, Jiyuan adjacent to Jiaozuo, Heze adjacent to Kaifeng and Shangqiu, and Huaibei adjacent to Shangqiu and Suzhou where Yuncheng exhibits the most obvious increase with absolute and relative changes in WATT values of $212.73 \mathrm{~min}$ and $31.28 \%$, respectively. From the perspective of the absolute and relative change of the WATT values, a city can enjoy a high absolute change and a significant improvement in relative accessibility, but due to its initially low levels, accessibility remains fairly low overall. 
Table 4. Weighted average travel time (WATT) changes observed for the CPER with and without HSR.

\begin{tabular}{|c|c|c|c|c|}
\hline \multirow[b]{2}{*}{ City } & \multicolumn{2}{|c|}{ WATT (Min) } & \multicolumn{2}{|c|}{ Change } \\
\hline & $\begin{array}{c}\text { Without HSR } \\
\text { Scenario }\end{array}$ & $\begin{array}{l}\text { With HSR } \\
\text { Scenario }\end{array}$ & Absolute Change (Min) & Relative Change (\%) \\
\hline Zhengzhou & 171.43 & 123.05 & 48.38 & 28.22 \\
\hline Kaifeng & 240.42 & 167.24 & 73.18 & 30.44 \\
\hline Luoyang & 260.14 & 164.17 & 95.97 & 36.89 \\
\hline Anyang & 281.81 & 182.14 & 99.67 & 35.37 \\
\hline Zhoukou & 314.46 & 296.66 & 17.81 & 5.66 \\
\hline Shangqiu & 254.96 & 167.94 & 87.01 & 34.13 \\
\hline Nanyang & 435.02 & 420.68 & 14.34 & 3.30 \\
\hline Xinyang & 306.19 & 184.20 & 121.99 & 39.84 \\
\hline Jiaozuo & 292.30 & 206.13 & 86.17 & 29.48 \\
\hline Jiyuan & 394.35 & 345.04 & 49.31 & 12.50 \\
\hline Luohe & 217.15 & 141.95 & 75.20 & 34.63 \\
\hline Xinxiang & 226.24 & 149.65 & 76.59 & 33.85 \\
\hline Pingdingshan & 317.72 & 300.14 & 17.58 & 5.53 \\
\hline Xuchang & 219.79 & 164.42 & 55.37 & 25.19 \\
\hline Zhumadian & 292.94 & 177.43 & 115.51 & 39.43 \\
\hline Hebi & 276.51 & 175.90 & 100.61 & 36.39 \\
\hline Puyang & 549.10 & 510.14 & 38.96 & 7.10 \\
\hline Sanmenxia & 369.23 & 211.58 & 157.65 & 42.70 \\
\hline Yuncheng & 680.07 & 467.34 & 212.73 & 31.28 \\
\hline Jincheng & 407.03 & 371.65 & 35.38 & 8.69 \\
\hline Changzhi & 512.58 & 476.64 & 35.94 & 7.01 \\
\hline Xingtai & 341.41 & 208.61 & 132.80 & 38.90 \\
\hline Handan & 320.18 & 198.91 & 121.26 & 37.87 \\
\hline Liaocheng & 367.43 & 332.05 & 35.38 & 9.63 \\
\hline Heze & 302.64 & 264.36 & 38.28 & 12.65 \\
\hline Huaibei & 457.32 & 328.03 & 129.30 & 28.27 \\
\hline Suzhou & 493.52 & 269.94 & 223.58 & 45.30 \\
\hline Bengbu & 442.55 & 288.97 & 153.58 & 34.70 \\
\hline Bozhou & 324.83 & 257.21 & 67.62 & 20.82 \\
\hline Fuyang & 303.99 & 273.44 & 30.55 & 10.05 \\
\hline Average & 345.78 & 260.85 & 84.93 & 24.56 \\
\hline
\end{tabular}

For typical cities, absolute and relative changes in accessibility are found to be greatest for Suzhou because before the opening of the HSR, the WATT value for Suzhou was high and the city's level of accessibility was low. The introduction of the HSR greatly improved Suzhou's accessibility levels by significantly reducing the city's travel times. Space-time convergence effects of the HSR are also found to be significant. It is worth noting that absolute and relative changes observed for Zhengzhou are less significant since before and after the introduction of the HSR, the WATT value for Zhengzhou was the lowest of the cities studied. Zhengzhou, the provincial capital city of Henan, serves as an important hub of the CPER transport network. Its level of accessibility was initially high, and the HSR further strengthened its role as a transportation hub. Accessibility levels remain relatively low before and after the introduction of HSR to cities such as Puyang and Nanyang. Improvements in accessibility from HSR are relatively minor for these cities, and they continue to experience relatively high absolute WATT values, which can be partly attributed to their relatively low level of economic development. Furthermore, railway connectivity levels in these cities are poor, and thus frequency distributions are not uniform. For cities such as Puyang, in which rail transport connecting to other cities is relatively uncommon, the efficiency of the transport network is greatly reduced. As a result, even with the HSR construction, diffusion effects are minor. 

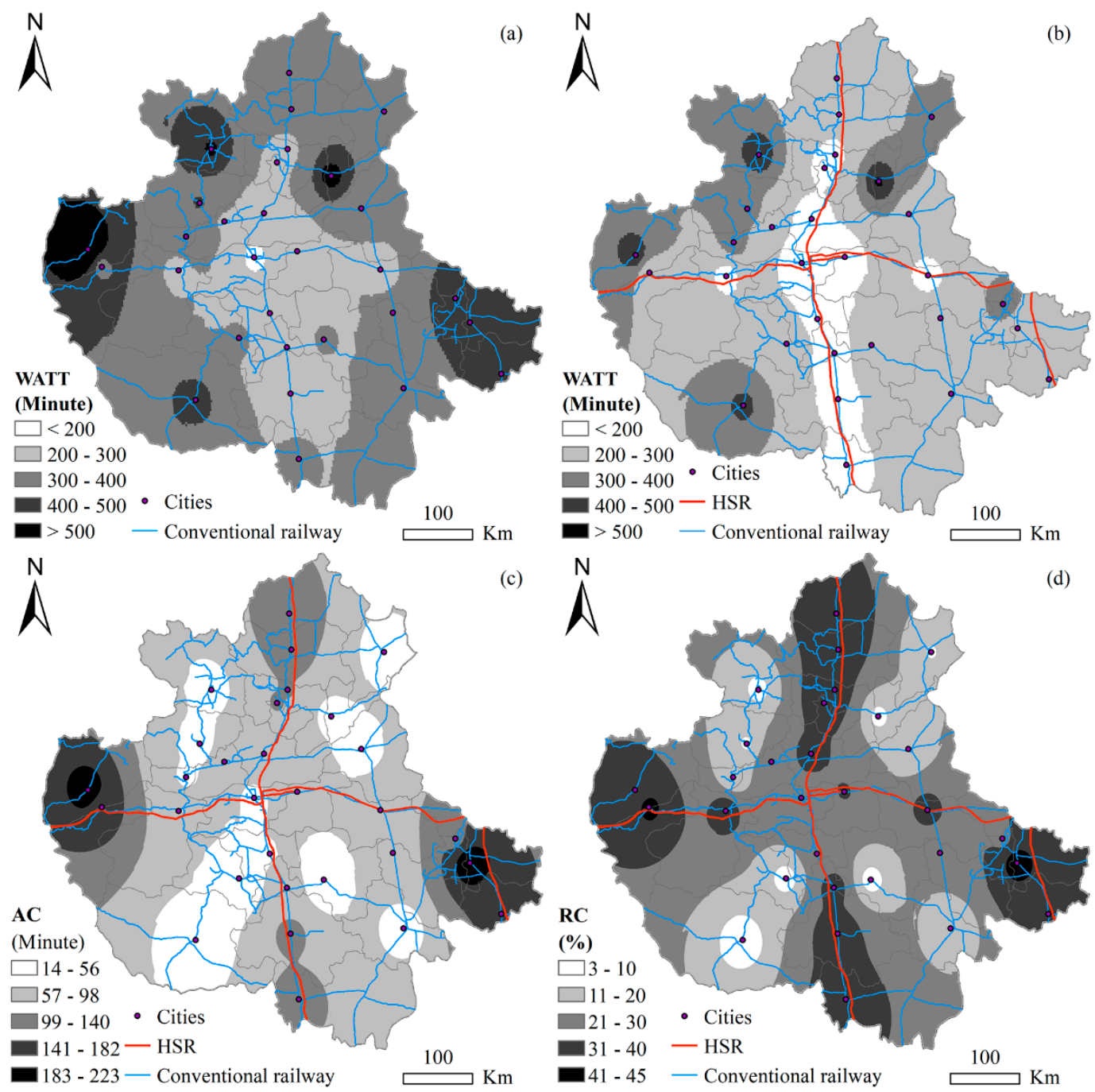

Figure 4. Spatial distribution of accessibility by WATT. Explanation for (a-d) here. (a) Without HSR Scenario WATT; (b) With HSR Scenario WATT (c) Absolute Change; (d) Relative Change.

\subsection{Equity Analysis}

$\mathrm{CV}$ values of the WATT for cities with and without HSR extensions were used as indicators to evaluate spatial discrepancy variability changes at the macro level. From the CV calculation results (Table 5), we find that CV values for all 30 cities increased from 0.32 to 0.41 , reflecting a $28.12 \%$ increase with HSR introduction. This implies that spatial differences of accessibility increased across cities. In the CPER, the level of disparity has not been relieved by travel time reduction.

Table 5. Coefficients of variance of (CV) changes in WATT for with and without HSR scenarios.

\begin{tabular}{cccc}
\hline & Without HSR Scenario & With HSR Scenario & Change (\%) \\
\hline Region & 0.32 & 0.41 & 28.12 \\
With HSR cities & 0.278 & 0.220 & -20.86 \\
Without HSR cities & 0.275 & 0.240 & -12.80 \\
Large cities & 0.279 & 0.338 & 21.01 \\
Small and medium-sized cities & 0.311 & 0.389 & 25.08 \\
\hline
\end{tabular}

For HSR and non-HSR cities, CV values of WATT for HSR cities fell by $20.86 \%$, and those for non-HSR cities fell by $12.80 \%$, showing that the discrepancy in WATT across HSR cities along the HSR corridor was reduced, and the accessibility discrepancy of non-HSR cities was also reduced in 
the CPER. The disparity observed in WATT values for non-HSR cities underwent a relatively minor decrease. Clearly, from the introduction of the HSR network, while the accessibility level improved, high accessibility values were concentrated in HSR cities, and differences among non-HSR cities became much more obvious. Contributors to overall spatial discrepancy in the CPER were mainly found to be non-HSR cities. Certain cities, such as Puyang, started to face higher levels of inequity than before the HSR introduction. Under the influence of the HSR system coupled with the divergence of locations, resource endowment, and geographical proximity, polarization was enhanced, trickle-down effects did not appear, and the Matthew effect (strengthening the strong and weakening the weak) [49] was enhanced significantly. In terms of city sizes, CV values for large cities increased by $21.01 \%$ and those for small and medium-sized cities increased by $25.08 \%$, showing that the HSR construction further widened disparities in WATT values within large cities and within small and medium-sized cities in the CPER. The disparity in WATT values observed within small and medium-sized cities increased more significantly. Apparently, after the introduction of an HSR network, the level of spatial equity is high if only large cities. Disparities across small and medium-sized cities become more notable than those of large cities. Spatial discrepancies observed in the CPER are mainly attributable to small and medium-sized cities.

\section{Discussion and Conclusions}

\subsection{Searching for Upgraded CR Lines}

Enhancing "flow" between cities and regions and improving the accessibility of regions are the most direct motivations for the implementation of a new HSR service [50]. However, cities located far from an HSR network can suffer from backwash effects and fall into a disadvantaged position [51]; the HSR operation enhances communication between regions but does not address the primary goal of mitigating regional disparities in accessibility [41]. Some regions affected by limited traffic networks suffer from spatial-equity disparities due to improvements made in other cities with strong economies and dense populations [2]. There are two ways to mitigate regional disparities: Infrastructure provision and the instalment of a suitable regulatory system with accompanying local measures under government supervision [41].

This study focuses on upgrading CR lines and building an "efficiency-equity" model to identify an optimized upgraded line to improve the overall efficiency of the CPER and to limit regional disparities. First, according to screening conditions, we screened candidate CR lines that can be upgraded to HSR lines such as those in Zhengzhou-Zhoukou, Xinyang-Nanyang, Jiaozuo-Xinxiang, Sanmenxia-Yuncheng and so on. Second, the shortest travel time between two cities was used according to the two models. Then, according to Equations (1) and (2), a new WATT and CV values were calculated. Finally, the most efficient and equitable CR line was identified from the calculated results. From the screening and calculations, in terms of upgrading just one line of $C R$, we found the CR line with the maximum improvement values on WATT and CV among all 119 sections of CR lines to be that of Xinxiang-Jiaozuo. By upgrading this CR line, the CV value decreased from 0.41 to 0.35 and then to the maximum value at a rate of $14.63 \%$ and has also reduced spatial differences in accessibility. In terms of upgrading two lines of CR, among the $119 \times 118 / 2$ candidate CR lines, we found the CR line with the maximum improvement values on WATT and CV to be that of "Xinxiang-Jiaozuo-Sanmenxia-Yuncheng". By upgrading these two CR lines, CV has decreased from 0.41 to 0.31 , at a rate of $24.39 \%$. In turn, accessibility level in the CPER improved significantly and differences in accessibility distribution in the CPER have been reduced, and regional levels of traffic integration have been enhanced.

\subsection{Comparisons of Relevant Research}

This paper extends studies on HSR efficiency and spatial equity. It adopts accessibility and spatial equity indicators derived from previous studies such as the WATT and CV, constructs an 
"efficiency-equity" model based on the upgrading of CR, and identifies the most important CR lines to upgrade to improve efficiency and constraint regional disparities. The findings of this study support the results of other relevant HSR literature in the sense that the HSR network generally improves accessibility $[1,10,12,14]$, but the degree of improvement varies (Table 4). This paper extends the work of relevant studying $[2,5,16,19,21,43]$ by searching for upgraded $C R$ lines. This study not only measured the spatial equality of accessibility (understand the world), but also tried to finish off the issues of efficiency and equity (reform the world). The results of this study serve as supportive information for the planning and construction of the HSR network and as a reference for the balancing of efficiency and equity in HSR construction, and can be used to facilitate underdeveloped regional transport system policy-making.

However, this study has limitations. First, as is the case for most previous studies, the accessibility level was only observed for CR and HSR systems without comprehensively considering highways, national and provincial roads, and other transport modes [52,53]. In practice, residents consider a variety of factors when selecting the most suitable mode of transport. Second, many indicators for evaluating accessibility are available $[5,46,47]$, while the WATT was measured only in terms of travel time savings in this study. In future studies, we can contribute to potential accessibility and daily accessibility indexes by analysing different factors. Third, many indexes for evaluating spatial equity are available, such as the social demand index, the GINI coefficient, the Theil indices and the Atkinson [16,34], Therefore, future research needs to consider these indexes.

\subsection{Future Research Directions}

Conventional train services have been adjusted since HSR adoption to avoid duplicating services, which causes accessibility to decline. An interesting issue regarding the advent of HSR is how to adjust conventional train frequency or how much conventional train services could be reduced while maintaining the accessibility level, which may also be an important way to improve regional accessibility and spatial equity. This question should be explored further in future research. Many studies on transport equity are largely focused on two dimensions: Horizontal and vertical [29]. While the latter dimension focuses on how accessibility distributes to specific populations equally, scholars have largely identified the former dimension as the uniform spatial distribution of accessibility levels [32], which is explored in this study. Research focusing on evaluating public transportation services in terms of equity is more heavily focused on vertical equity or on relative levels of service quality that benefit transportation-disadvantaged people [54,55]. Issues of horizontal and vertical equity should be explored in future works.

\subsection{Conclusions}

Based on the perspective of efficiency and spatial equity, this paper uses the WATT and CV to measure the impact of HSR on efficiency and equity in the CPER and to build an "efficiency-equity" model to optimally upgrade CR lines to improve the efficiency of the CPER and to decrease regional disparities. The major findings of this study are summarized as follows:

(1) In terms of efficiency levels, with the HSR introduction, the accessibility of the CPER was significantly enhanced overall. HSR has obvious space-time convergence effects, shortening travel time between cities, improving the accessibility of the CPER, and enhancing "corridor effects" along the HSR. Cities showing a greater absolute and relative increase in accessibility are distributed along the "HSR corridor".

(2) From an equity perspective, disparities in regional accessibility increased due to the HSR, and the spatial equity of the CPER generally declined. The construction of the HSR further mitigated disparities in the WATT within both HSR cities and non-HSR cities. Equitable accessibility has mainly been concentrated in HSR cities, and non-HSR cities have been the main contributors to overall spatial discrepancies in the CPER. The construction of HSR further exacerbated disparities in the WATT within large and within small and medium-sized cities. The level of spatial equity is high if only large cities, 
and disparities across small and medium-sized cities are much more notable, showing that small and medium-sized cities have mainly contributed to spatial discrepancies in the CPER.

By taking both "efficiency" and "equity" as goals and the upgrading of CR lines as a new approach, this study found that "Xinxiang-Jiaozuo" and "Xinxiang-Jiaozuo-Sanmenxia-Yuncheng" are optimal upgrading lines for improving accessibility and mitigating regional disparities in the CEPR.

Author Contributions: Writing-Original Draft Preparation, X.W.; Writing—Review and Editing, W.Z.

Funding: This research was funded by China Social Welfare Foundation, grant number ZJ20150129.

Conflicts of Interest: The authors declare no conflict of interest.

\section{Appendix A}

Table A1. Comparing the contributions of some literature.

\begin{tabular}{|c|c|}
\hline Representative Author, Year & Contributions in Approach and Results \\
\hline Gutiérrez, J. 1996, 2001, 2010 & $\begin{array}{l}\text { Use Weighted average travel time; Economic potential and Daily } \\
\text { accessibility indicators; HSR can improve city accessibility levels and } \\
\text { changing regional spatial structure. }\end{array}$ \\
\hline López, S. 2008 & $\begin{array}{l}\text { Comprehensive use accessibility and spatial equity indicators: Location; } \\
\text { Potential; Network efficiency; Daily accessibility; CV; GINI coefficient; } \\
\text { Theil indices; Atkinson. }\end{array}$ \\
\hline Garmendia, M. 2011 & $\begin{array}{l}\text { Assess the impact of high-speed infrastructures from the perspective of } \\
\text { commuting and discretionary flows; HSR can improve the accessibility } \\
\text { and location advantages of sparsely populated or isolated areas and } \\
\text { enhance regional equity. }\end{array}$ \\
\hline Ortega, E. 2012 & $\begin{array}{l}\text { Evaluate cohesion impacts from different planning levels: regional, } \\
\text { corridor and national levels; The national and corridor levels appear } \\
\text { positive cohesion effects, but region appear polarization effects; some } \\
\text { policy measures are suggested. }\end{array}$ \\
\hline Levinson, D. 2012 & $\begin{array}{l}\text { Small and medium-sized cities may achieve absolute accessibility and } \\
\text { lose in relative position. }\end{array}$ \\
\hline Monzón, A. 2013 & Assess the magnitude and distribution of the accessibility. \\
\hline Kim, M. 2015 & $\begin{array}{l}\text { Assess the accessibility of each stage of HSR network; Accessibility } \\
\text { improvement has concentrated in the primary HSR corridor cities. }\end{array}$ \\
\hline Wang, L. 2018 & $\begin{array}{l}\text { Added territorial and population accessibility to measure spatial equity; } \\
\text { HSR has not decreased the disparity in territorial accessibility but } \\
\text { decrease the disparity in population accessibility. }\end{array}$ \\
\hline Yang, J. 2018 & $\begin{array}{l}\text { Added social demand index to measure spatial equity; } \\
\text { Form "corridors" and "islands". }\end{array}$ \\
\hline Monzón, A. 2019 & $\begin{array}{l}\text { Assess "accessibility-based" territorial cohesion effects of HSR corridors } \\
\text { in 1990-2015 period; At the beginning of the construction of the HSR } \\
\text { network, efficiency was prioritized; Need to integrate efficiency and } \\
\text { Spatial equity. }\end{array}$ \\
\hline
\end{tabular}

\section{References}

1. Maækiewicz, A.; Ratajczak, W. Towards a new definition of topological accessibility. Transp. Res. B Methodol. 1996, 30, 47-79. [CrossRef]

2. Kim, M.; Sultana, S. The impacts of high-speed rail extensions on accessibility and spatial equity changes in South Korea from 2004 to 2018. J. Transp. Geogr. 2015, 45, 48-61. [CrossRef]

3. Hansen, W.G. How accessibility shapes land use. J. Am. Inst. Plan. 1959, 25, 73-76. [CrossRef] 
4. Adler, N.; Pels, E.; Nash, C. High-speed rail and air transport competition: Game engineering as tool for cost-benefit analysis. Transp. Res. B Methodol. 2010, 44, 812-833. [CrossRef]

5. Monzón, A.; Ortega, E.; López, E. Efficiency and spatial equity impacts of high speed rail extensions in urban areas. Cities 2013, 30, 18-30. [CrossRef]

6. Miao, Y.; Wei, F. Accessibility impact of future high speed rail corridor on the piedmont Atlantic megaregion. J. Transp. Geogr. 2018, 73, 1-12.

7. Liu, L.W.; Zhang, M. High-speed rail impacts on travel times, accessibility, and economic productivity: A benchmarking analysis in city-cluster regions of China. J. Transp. Geogr. 2018, 73, 25-40. [CrossRef]

8. Hou, Q.; Li, S.M. Transport infrastructure development and changing spatial accessibility in the Greater Pearl River Delta, China, 1990-2020. J. Transp. Geogr. 2011, 19, 1350-1360. [CrossRef]

9. Chen, H.S.; Sun, D.Q.; Zhu, Z.J.; Zeng, J. The Impact of High-Speed Rail on Residents' Travel Behavior and Household Mobility: A Case Study ofthe Beijing-Shanghai Line, China. Sustainability 2016, 8, 1187. [CrossRef]

10. Wang, L. High-speed rail services development and regional accessibility restructuring in megaregions: A case of the Yangtze River Delta, China. Transp. Policy 2018, 72, 34-44. [CrossRef]

11. Bröcker, J.; Korzhenevych, A.; Schürmann, C. Assessing spatial equity and efficiency impacts of transport infrastructure projects. Transp. Res. B Methodol. 2010, 44, 795-811. [CrossRef]

12. Gutiérrez, J.; Condeço-Melhorado, A.; Martín, J.C. Using accessibility indicators and GIS to access spatial spillovers of transport infrastructure investment. J. Transp. Geogr. 2010, 18, 141-152. [CrossRef]

13. Shaw, S.L.; Fang, Z.; Lu, S.; Tao, R. Impacts of high speed rail on railroad network accessibility in China. J. Transp. Geogr. 2014, 40, 112-122. [CrossRef]

14. Wang, L.H.; Liu, Y.X.; Sun, C.; Liu, Y.H. Accessibility impact of the present and future high-speed rail network: A case study of Jiangsu Province, China. J. Transp. Geogr. 2016, 54, 161-172. [CrossRef]

15. Hay, A. Equity and welfare in the geography of public transport provision. J. Transp. Geogr. 1993, 1, 95-101. [CrossRef]

16. Yang, J.; Guo, A.D.; Li, X.M.; Huang, T. Study of the Impact of a High-Speed Railway Opening on China's Accessibility Pattern and Spatial Equality. Sustainability 2018, 10, 2943. [CrossRef]

17. Levinson, D. Accessibility impacts of high-speed rail. J. Transp. Geogr. 2012, 22, 288-291. [CrossRef]

18. Cao, J.; Liu, X.Y.; Wang, Y.H.; Li, Q.Q. Accessibility impacts of China's high-speed rail network. J. Transp. Geogr. 2013, 28, 12-21. [CrossRef]

19. Ortega, E.; López, E.; Monzón, A. Territorial cohesion impacts of high-speed rail at different planning levels. J. Transp. Geogr. 2012, 24, 130-141. [CrossRef]

20. Martínez Sánchez-Mateos, H.S.; Givoni, M. The accessibility impacts of a new High-Speed Rail line in the UK-A preliminary analysis of winners and losers. J. Transp. Geogr. 2012, 25, 105-114. [CrossRef]

21. Wang, L.; Duan, J.D. High-speed rail network development and winner and loser cities in megaregions: The case study of Yangtze River Delta, China. Cities 2018, 83, 71-82. [CrossRef]

22. Gutiérrez, J.; Gonzalez, R.; Gomez, G. The European high-speed train network: Predicted effects on accessibility patterns. J. Transp. Geogr. 1996, 4, 227-238. [CrossRef]

23. Martin, J. Justifying a high-speed rail project: Social value vs. regional growth. Ann. Reg. Sci. 1997, 31, 155-174. [CrossRef]

24. Javier, G. Location, economic potential and daily accessibility: An analysis of the accessibility impact of the high-speed line Madrid-Barcelona-French border. J. Transp. Geogr. 2001, 9, 229-242.

25. Long, F.J.; Zheng, L.F.; Song, Z.D. High-speed rail and urban expansion: An empirical study using a time series of nighttime light satellite data in China. J. Transp. Geogr. 2018, 72, 106-118. [CrossRef]

26. Páez, A.; Scott, D.M.; Morency, C. Measuring accessibility: Positive and normative implementations of various accessibility indicators. J. Transp. Geogr. 2012, 25, 141-153. [CrossRef]

27. Ureña, J.M.; Menerault, P.; Garmendia, M. The high-speed rail challenge for big intermediate cities: A national, regional and local perspective. Cities 2009, 26, 266-279. [CrossRef]

28. Martens, K.; Golub, A.; Robinson, G. A justice-theoretic approach to the distribution of transportation benefits: Implications for transportation planning practice in the United States. Transp. Res. A Policy Pract. 2012, 46, 684-695. [CrossRef]

29. Litman, T. Evaluating transportation equity. World Transp. Policy Pract. 2002, 8, 50-65.

30. Caggiani, L.; Camporeale, R.; Michele, O. Facing equity in transportation Network Design Problem: A flexible constraints based model. Transp. Policy 2017, 55, 9-17. [CrossRef] 
31. Chang, H.S.; Liao, C.H. Exploring an integrated method for measuring the relative spatial equity in public facilities in the context of urban parks. Cities 2011, 28, 361-371. [CrossRef]

32. Thomopoulos, N.; Grant-Muller, S.; Tight, M.R. Incorporating equity considerations in transport infrastructure evaluation: Current practice and a proposed methodology. Eval. Progr. Plan. 2009, 32, 351-359. [CrossRef] [PubMed]

33. El-Geneidy, A.; Levinson, D.; Diab, E.; Boisjoly, G.; Verbich, D.; Loong, C. The cost of equity: Assessing transit accessibility and social disparity using total travel cost. Transp. Res. A Policy Pract. 2016, 91, 302-316. [CrossRef]

34. López, S.; Gutiérrez, J.; Gómez, G. Measuring regional cohesion effects of large-scale transport infrastructure investments: An accessibility approach. Eur. Plan. Stud. 2008, 16, 277-301. [CrossRef]

35. Domanski, R. Accessibility, efficiency and spatial organization. Environ. Plan. 1979, 11, 1189-1206. [CrossRef]

36. Verma, A.; Sudhira, H.S.; Rathi, S.; King, R.; Dash, N. Sustainable urbanization using high speed rail (HSR) in Karnataka, India. Res. Transp. Econ. 2013, 38, 67-77. [CrossRef]

37. Hall, P. Magic carpets and seamless webs: Opportunities and constraints for high-speed trains in Europe. Built Environ. 2009, 35, 59-69. [CrossRef]

38. Garmendia, M.; Ureña, J.M.; Coronado, J.M. Long-distance trips in a sparsely populated region: The impact of high-speed infrastructures. J. Transp. Geogr. 2011, 19, 537-551. [CrossRef]

39. Chen, Z.H. Impacts of high-speed rail on domestic air transportation in China. J. Transp. Geogr. 2017, 62, 184-196. [CrossRef]

40. Albalate, D.; Bel, G.; Fageda, X. Competition and cooperation between high-speed rail and air transportation services in Europe. J. Transp. Geogr. 2015, 42, 166-174. [CrossRef]

41. Vickerman, R. High-speed rail and regional development: The case of intermediate stations. J. Transp. Geogr. 2015, 42, 157-165. [CrossRef]

42. Mohino, I.; Loukaitou-Sideris, A.; Urena, J.M. Impacts of high-speed rail on metropolitan integration: An examination of London, Madrid and Paris. Int. Plan. Stud. 2014, 19, 306-334. [CrossRef]

43. Monzón, A.; López, E.; Ortega, E. Has HSR improved territorial cohesion in Spain? An accessibility analysis of the first 25 years: 1990-2015. Eur. Plan. Stud. 2019, 27, 513-532. [CrossRef]

44. Wu, J.; Nash, C.; Wang, D. Is high speed rail an appropriate solution to China's rail capacity problems? J. Transp. Geogr. 2014, 40, 100-111. [CrossRef]

45. Fan, J.; Li, P.X. The scientific foundation of Major Function Oriented Zoning in China. Geogr. Sci. 2009, 5, 515-531. [CrossRef]

46. Wu, W.; Liang, Y.; Wu, D. Evaluating the Impact of China's Rail Network Expansions on Local Accessibility: A Market Potential Approach. Sustainability 2006, 8, 512-613. [CrossRef]

47. Jiao, J.; Wang, J.; Jin, F.; Dunford, M. Impacts on accessibility of China's present and future HSR network. J. Transp. Geogr. 2014, 40, 123-132. [CrossRef]

48. Wang, L.H.; Liu, Y.X.; Mao, L.; Sun, C. Potential Impacts of China 2030 High-Speed Rail Network on Ground Transportation Accessibility. Sustainability 2018, 10, 1270. [CrossRef]

49. Wan, Y. The Matthew Effect in social commerce. Electron. Mark. 2015, 25, 313-324. [CrossRef]

50. Blum, U.; Haynes, K.E.; Karlsson, C. Introduction to the special issue: The regional and urban effects of high-speed trains. Ann. Reg. Sci. 1997, 31, 1-20. [CrossRef]

51. Biggiero, L.; Pagliara, F.; Patrone, A.; Peruggini, F. Spatial equity and high-speed rail systems. Int. J. Transp. Dev. Integr. 2017, 2, 194-202. [CrossRef]

52. Meng, D.Y.; Lu, Y.Q. Impact of high-speed railway on accessibility and economic linkage of cities along the railway in Henan province, China. Sci. Geogr. Sin. 2011, 31, 537-543.

53. Yang, J.H. Study on the regional accessibility of urban agglomeration in the HuNan province under the impact of High-speed railway. Hum. Geogr. 2016, 2, 108-112.

54. Delbosc, A.; Currie, G. Using Lorenz curves to assess public transport equity. J. Transp. Geogr. 2011, 19, 1252-1259. [CrossRef]

55. Foth, N.; Manaugh, K.; El-Geneidy, A.M. Towards equitable transit: Examining transit accessibility and social need in Toronto, Canada, 1996-2006. J. Transp. Geogr. 2013, 29, 1-10. [CrossRef]

(C) 2019 by the authors. Licensee MDPI, Basel, Switzerland. This article is an open access article distributed under the terms and conditions of the Creative Commons Attribution (CC BY) license (http://creativecommons.org/licenses/by/4.0/). 